Sakurai, T. 1981, Solar Phys., 769, 343.

Sakurai, T., and Granik, A. 1981, Ap. J., 277, 404.

Sakurai, T., and Levine, R.H. 1981, Ap. J., $248,817$.

Schindler, K., Birn, J., and Janicke, L. 1983, Solar Phys., 87, 103.

Schwartz, S.J., and Bel, N. 1984a, Astr. Ap., 137, 128.

Schwartz, S.J., and Bel, N. 1984b, Solar Phys., 92, 133.

Schwartrz, S.J., Cally, P.S., and Bel, N. 1984, Solar Phys., 92, 81.

Schwartz, S.J., and Leroy, B. 1982, Astr. Ap., 112, 93.

Serio, S., Peres, G., Vaiana, G.S., Golub, L., and Rosner, R. 1981, Ap. J., 243, 288.

Spicer, D.S. 1981, Solar Phys., 71, 115.

Spicer, D.S. 1982, Space Sci. Rev., 31, 351.

Spruit, H.C. 1981, in The Sun as a Star, ed. S. Jordan (Washington: NASA SP-450), p. 385.

Spruit, H.C. 1982, Solar Phys., 75, 3.

Spruit, H.C., and Roberts, B. 1983, Nature, 304, 401.

Steinolfson, R.S. 1984, Phys. Fluids, 27, 781.

Strauss, F.M., Kaufmann, P., and Opher, R. 1980, solar Phys., 67, 83.

Sturrock, P.A., and Uchida, Y. 1981, Ap. J., 246, 331.

Takakura, T., Kaufmann, P., Costa, J.E.R., Degaonkar, S.S., Ohki, K., and Nitta, N. 1983, Nature, $302,317$.

Taylor, J.B. 1974, Phys. Rev. Letters. 33, 1139.

Thomas, J.H. 1983, Ann. Rev. Fluid Mech., 15, 321.

Torricelli-Ciamponi, G., Einaudi, G., and Chiuderi, C. 1982, Astr. Ap., 105, L1.

Trottet, G., Kerdraon, A., Benz, A.O., and Treymann, R. 1981, Astr. Ap., 93, 129.

Tsinganos, K.C. 1982, Ap. J., 259, 832.

Van Hoven, G. 1981, in Solar Flare Magnetohydrodynamics, ed. E.R. Priest (New York: Gordon and Breach), p. 217.

Van Hoven, G., Ma, S., and Einaudi, G. 1981, Astr. Ap., 99, 232.

Wentzel, D.G. 1979, Astr. Ap., 76, 20.

Wilson, P.R. 1980, Astr. Ap., 87, 121.

Withbroe, G. 1981, in Solar Active Regions, ed. F. Orrall (Boulder: Colorado Assoc. Univ. Press).

Wragg, M.A., and Priest, E.R. 1981, Solar Phys., 70, 293.

Wragg, M.A., and Priest, E.R. 1982, Astr. Ap., 113, 269.

Zhugzhda, Y.D., and Dzhalilov, N.S. 1984, Astr. Ap., 132, 45, 52.

Zhugzhda, Y.D., and Locans, V. 1982, Solar Phys., 76, 77.

Zweibel, E.G., and Hundhausen, A. 1982, Solar Phys., 76, 261.

\title{
VII. THE SUN AS A STAR
}

\section{(K. R. Sivaraman)}

An important topic of current interest both in solar and stellar physics is the possibility of identifying solar phenomena in stars through an understanding of these on a global scale on the sun. We concentrate here on the recent research concerning the variability in solar irradiance from disc integrated observations (sun as a star) in the white light as well as in monochromatic wavelengths.

\section{Luminosity variation in white light}

The principal scientific motivation for monitoring the solar constant is to detect any variability that could explain the climate changes over the earth. For an excellent review see Newkirk (1983). Measurements in 1980 by the precision radiometers on NIMBUS-7 and SMM have revealed temporal dips of 0.1 to $0.3 \%$ in the measured solar irradiance which had eluded previous detection. An empirical model ascribing $90 \%$ of the short time irradiance fluctuations to sunspot blocking reproduces best the ACRIM data between 1980-1982. The solar irradiance reconstructed from the archived sunspot areas from 1874 through 1981 using this model imply that the average solar constant during an active year can be as much as $0.1 \%$ lower than during a quiet year (Hoyt and Eddy, 1983). Photospheric heat flow can also be modulated by large laminar convective cells causing temperature perturbations between up and down flowing regions on scales of $\sim 1 \times 10^{5} \mathrm{~km}$ and life times of months (Gilman, 1978). Also the subsurface magnetic concentrations might cause 'thermal shadows' on the photosphere (Spruit, 1977; Foukal et al. 1983). But the continuum maps outside spots and faculae show that temperature inhomogeneities on these scales are below $2-3^{\circ} \mathrm{K}$, which is far below the spot signal (Foukal and Fowler, 1984). The heat blocked by 
sunspots is apparently rapidly redistributed for storage throughout the convection zone over time scales at least comparable to the spot life times. The efficient storage and slow release explains the spot induced dips in the radiometry data. (Foukal et al. 1983).

\section{Luminosity variations in monochromatic radiation}

\subsection{Photospheric variability}

Changes in luminosity are intimately related to the possible slow global changes in photospheric temperature which can be detected by monitoring the equivalent widths of temperature sensitive spectral lines originating in the photosphere. During the ascending phase of solar activity (1976-1980) the full disc equivalent widths of CI 5380A and four $\mathrm{Fe} I$ lines representing the low and high photosphere showed a steady decrease, but remained constant since 1980 (Livingston and Holweger, 1982; Holweger et al. 1983). This calls for more data to decide the presence of any 11-year cycle modulation. Similar behaviour of all the diagnostic lines can be reconciled only by a slight flattening of the photospheric temperature gradient as the carbon line has a temperature sensitivity opposite to that of iron lines. Since the temporal behaviour of these lines has no correlation with short lived features like active regions, it seems to reflect true global changes accompanying solar activity.

Another diagnostic is the spectral line asymmetry which is a measure of the granular convection. The C-shaped line bisector is more blue shifted relative to the core over quiet regions than in magnetic regions. The full disc Fe I 5250.6 mean bisectors from the Kitt Peak $13.5 \mathrm{~m}$ spectrograph show a mid-C shift towards the core by $\sim 2 \mathrm{~mA}$ between 1976 and 1981 . Better observations with the FTS of ten similar Fe I lines confirm this trend between 1980-1982, suggesting a global decrease in the net upward velocity of convection in the photosphere with increase in solar activity (Livingston, 1982). Pic du Midi observations showing that the size of granules is reduced at that time support this (Macris and Rösch, 1983). Far infrared radiometry of the full disc indicates a warming of the temperature minimum accompanying solar activity (Müller et al. level in the atmosphere contradicts this finding (Cook et al. 1980).

\subsection{Chromospheric variability}

$K$-emission. The first attempt to demonstrate the variability in the $\mathrm{Ca}$ II $\mathrm{K}$ line emission from the 'Sun as a star' was by Bumba and Topolova (1967). The continuing disc in tegrated K emission measurements commencing in 1969 at Kodaikanal provide data on the variability of the K-line parameters over a full cycle (Bappu and Sivaraman, 1971; Sivaraman et al. 1984). Other published results, although for a limited period are those of White and Livingston (1978) and Kei] and Worden (1984). The 1A emission index for the years 1975-1981 suggest that the excess emission in the ascending phase of the solar cycle arises from plages (White and Livingston, 1978). The quiet sun is represented well by the summation of the contributions from the network and the cell interior forming one component. The addition of two or more components, the plages and the active network (remnants of decayed plages) gives good agreement with full disc emission except near the solar maximum (Skumanich et al. 1984).

He I 10890A. Full disc He I 10830A equivalent width measurements since 1974 show it was lowest at $24 \mathrm{~mA}$ in mid 1975 , a year earlier than sunspot minimum and steadily rose afterwards to $83 \mathrm{~mA}$ in late 1981, two years after the sunspot minimum. The rotational modulation reveals active longitudes. The apparent rotation period increased from 1977 to 1981 and has been shorter since 1981 which contradicts the equatorial migration of active regions during the course of solar cycle (Harvey, 1983).

Lyman Alpha. The ground based $\mathrm{K}$ index serves as a good proxy to space measures of Ly $\alpha$ and the EUV. The Ly $\alpha$ flux computed using a three component model of $\mathrm{K}$ with the photometric contrasts at Ly $\alpha$ agrees well with the measured flux in the years 1969-1980 except near the solar maximum. High resolution observations may bring better agreement as, at 1 " $\times 1$ " resolution $33 \%$ of the disc would have enhanced Ly $\alpha$ emission at solar maximum (Lean and Skumanich, 1983).

\section{Radius variation}

A change in the solar output would require a change in the solar radius. A day to day variation of 0.4" is detected by the dedicated solar diameter telescope in Boulder (Brown 1982). LaBonte and Howard (1981) from analysis of 5 years magnetograph data report that $R$ is constant to within \pm 0.1 ". The main problem is to be able to relate any apparent diameter change to change in lumi- 
nosity with certainty. To what accuracy must $\Delta R / R$ be measured to produce a change of $\Delta \mathrm{L} / \mathrm{L} \sim 0.1 \%$ ? Unless this is settled, the results of the radius change measurements cannot be linked to luminosity changes.

4. Rotation

A plot of the daily mean plage areas would reveal the 27 day modulation (Bappu and Sivaraman, 1971). Temporal power spectra of the daily plage index for the period 1976-1982 as a whole as well as of the broken data of one year each show only two peaks at 27 day and 13 day periods, (Keil and Worden, 1984). Analysis of the Mt. Wilson disc integrated magnetic flux also shows the 27 day and its harmonics with an accuracy of $\sim 2 \%$ (LaBonte, 1982). But none of the attempts have revealed the differential rotation of the Sun from full disc data.

5. Solar mean magnetic field

The only measurement of the magnetic field of the sun integrated over the entire disc is the one using the Fe I 5250A line for the years 1968-1981 due to Severny and his co-workers. Year to year values of the yearly means of the mean magnetic field show changes as much as by factors 3 to 4 (Kotov and Severny, 1983).

\section{References}

Bappu, M.K.V.; and Sivaraman, K.R.; 1971, Solar Phys. 17, 316.

Bappu, M.K.V.; and Sivaraman, K.R.; 1977, Mon. Not. R. Astr. Soc. 78, 279.

Brown, T.M.; Elmore, D.F.; Lacey, L.; and Hull, H.; 1982, Appl. Opt. 21, 3588.

Bumba, V.; and Topolova, B.R.; 1967, Solar Phys. 1, 216.

Cook, J.W.; Brueckner, G.E.; and Van Hoosier, M.E.; 1980, Astron. Astrophys. 92, L7.

Foukal, P.; Fowler, L.; and Livshits, M.; 1983, Astrophys. J. 267, 863

Foukal, P.; and Fowler, L.; 1984, Astrophys. J. 281, 442.

Gilman, P.; 1978, Geophys. Astrophys. Fluid Dyn. 11, 157.

Harvey, J.W.; 1983, in Workshop on 'Solar Variations on Active Region Time Scales' eds. Chapman, Hudson and LaBonte, Pasadena.

Holweger, H.; Livingston, W.; and Steenbock, W.; 1983, Nature, 302, 125.

Hoyt, D.V.; and Eddy, J.A.; 1983, Geophys. Res. Lett. 10, 509.

Keil, S.L.; and Worden, S.P.; 1984, Astrophys. J. 276, 766.

Kotov, V.A.; and Severny, A.B.; 1983, Materials of the World Data Centre B, Moscow.

LaBonte, B.J.; and Howard, R.; 1981, Science, $214,907$.

LaBonte, B.J.; 1982, Astrophys. J. 260, 647.

Lean, J.L.; and Skumanich, A.; 1983, Geophys. Res. 88, 5751.

Livingston, W.; 1982, Nature, 297, 208.

Livingston, W.; and Holweger,H.; 1982, Astrophys. J. 252, 375.

Macris, C.J.; and Rösch, J.; 1983, C.R. 296, 265.

Müller, E.A.; and Kneubühl, F.K.; Rast, J.; and Stettler, P.; 1980, Astron. Astrophys. 87, L3

Newkirk, Jr. G.; 1983, Ann Rev. Astron. Astrophys. $21,429$.

Sivaraman, K.R.; Jagdev Singh; Bagare, S.P.; and Gupta, S.S.; 1984, Astrophys. J. 282, 776.

Spruit, H.; 1977, Solar Phys. 55, 3.

White, O.R.; and Livingston, W.; 1978, Astrophys. J. 226, 679. 